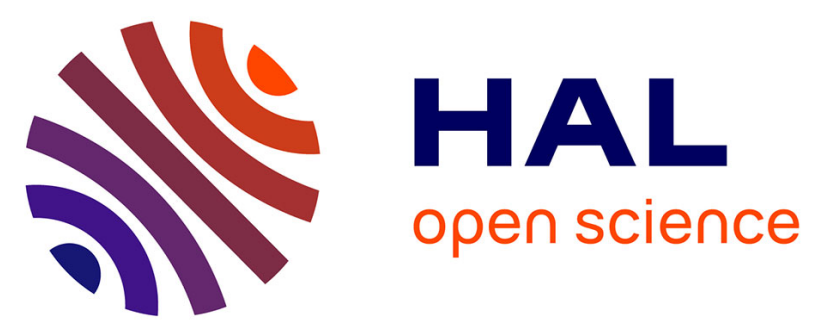

\title{
Dettes publiques et stabilité monétaire en Europe: les leçons de l'étalon or
}

\author{
Marc Flandreau, Jacques Le Cacheux
}

\section{To cite this version:}

Marc Flandreau, Jacques Le Cacheux. Dettes publiques et stabilité monétaire en Europe : les leçons de l'étalon or. Revue Economique, 1997, 48 (3), pp.529-538. 10.3406/reco.1997.409893 . hal-01027627

\section{HAL Id: hal-01027627 \\ https://hal-sciencespo.archives-ouvertes.fr/hal-01027627}

Submitted on 22 Jul 2014

HAL is a multi-disciplinary open access archive for the deposit and dissemination of scientific research documents, whether they are published or not. The documents may come from teaching and research institutions in France or abroad, or from public or private research centers.
L'archive ouverte pluridisciplinaire HAL, est destinée au dépôt et à la diffusion de documents scientifiques de niveau recherche, publiés ou non, émanant des établissements d'enseignement et de recherche français ou étrangers, des laboratoires publics ou privés.

\section{(이)(\$)}

Distributed under a Creative Commons Attribution - NonCommercial - NoDerivatives| 4.0 


\title{
Persée
}

http://www.persee.fr

\section{Dettes publiques et stabilité monétaire en Europe. Les leçons de l'étalon or.}

\author{
Marc Flandreau;Jacques Le Cacheux \\ Revue économique, Année 1997, Volume 48, Numéro 3 \\ p. $529-538$
}

Voir l'article en ligne

\section{Avertissement}

L'éditeur du site «PERSEE » - le Ministère de la jeunesse, de l'éducation nationale et de la recherche, Direction de l'enseignement supérieur, Sous-direction des bibliothèques et de la documentation - détient la propriété intellectuelle et les droits d'exploitation. A ce titre il est titulaire des droits d'auteur et du droit sui generis du producteur de bases de données sur ce site conformément à la loi n`98-536 du 1 er juillet 1998 relative aux bases de données.

Les oeuvres reproduites sur le site «PERSEE » sont protégées par les dispositions générales du Code de la propriété intellectuelle.

Droits et devoirs des utilisateurs

Pour un usage strictement privé, la simple reproduction du contenu de ce site est libre.

Pour un usage scientifique ou pédagogique, à des fins de recherches, d'enseignement ou de communication excluant toute exploitation commerciale, la reproduction et la communication au public du contenu de ce site sont autorisées, sous réserve que celles-ci servent d'illustration, ne soient pas substantielles et ne soient pas expressément limitées (plans ou photographies). La mention Le Ministère de la jeunesse, de l'éducation nationale et de la recherche, Direction de l'enseignement supérieur, Sous-direction des bibliothèques et de la documentation sur chaque reproduction tirée du site est obligatoire ainsi que le nom de la revue et- lorsqu'ils sont indiqués - le nom de l'auteur et la référence du document reproduit.

Toute autre reproduction ou communication au public, intégrale ou substantielle du contenu de ce site, par quelque procédé que ce soit, de l'éditeur original de l'oeuvre, de l'auteur et de ses ayants droit.

La reproduction et l'exploitation des photographies et des plans, y compris à des fins commerciales, doivent être autorisés par l'éditeur du site, Le Ministère de la jeunesse, de l'éducation nationale et de la recherche, Direction de l'enseignement supérieur, Sous-direction des bibliothèques et de la documentation (voir http://www.sup.adc.education.fr/bib/ ). La source et les crédits devront toujours être mentionnés. 


\title{
Dettes publiques et stabilité monétaire en Europe
}

Les leçons de l'étalon or

\author{
Marc Flandreau * \\ Jacques Le Cacheux **
}

\begin{abstract}
Dans cet article, nous mettons en évidence que l'étalon or se présente comme une gigantesque accumulation de dettes publiques et nous dégageons plusieurs hypothèses relatives aux facteurs réels et monétaires ayant permis cette accumulation. En second lieu, en nous concentrant sur les "pathologies" de l'étalon or, nous insistons sur les motifs systémiques et nationaux ayant facilité le développement ou au contraire la résolution de ces difficultés.
\end{abstract}

\section{PUBLIC DEBTS AND MONETARY STABILITY IN EUROPE : LESSONS FROM THE GOLD STANDARD}

In this paper, we show that the European gold standard 1880-1914 was characterized by a considerable accumulation of public debts, and we point to both real and monetary factors that may have accounted for this feature. Second, by concentrating on various pathological episodes, we argue that the stability of exchange rates resulted from a mix of systemic forces and institutional arrangements, which mitigated, or instead amplified national difficulties.

Classification JEL : F02, F33, F34, N13

\section{INTRODUCTION}

Cet article constitue une sorte de rapport intermédiaire dans un projet de recherche commencé à l'OFCE depuis l'été 1995 et visant à renouveler, au travers de la collecte et de la constitution de nouvelles séries, le débat sur les fondements de la stabilité de l'étalon or, avant 1914. Notre point de départ était la tentative de répondre à la question, rituelle chez les chercheurs en économie monétaire internationale, du « miracle » de la stabilité de l'étalon or, entre 1880 et 1914. Si elle a été souvent débattue, cette question revêt un intérêt particulier dans le cadre européen actuel, puisqu'il s'agit aujourd'hui encore de fonder le développement économique du continent sur la création d'une zone monétaire homogène.

\footnotetext{
* CNRS, et OFCE, 69 quai d'Orsay, 75340 Paris Cedex 07.

** Université de Pau et des Pays de l'Adour et OFCE.
} 
Dans ce programme de recherche, notre ambition est double. D'un côté, il s'agit d'éclairer les évolutions actuelles sous l'angle de la longue période. En effet, dans l'esprit de la majorité des économistes, l'étalon or décroche sans nul doute un «prix de vertu ». Il fournit donc une sorte de point de repère « orthodoxe» par rapport auquel on peut juger de la situation présente. En second lieu, il s'agit pour nous d'enrichir l'étude du fonctionnement de la zone or avant 1914. En particulier, nous voulions, au travers de ce travail, dépasser le cadre excessivement « anglo-centrique » dans lequel la discussion de la stabilité de l'étalon or est habituellement conduite ${ }^{1}$. Placée sous un éclairage global, la situation britannique apparaît bien plus originale qu'exemplaire ${ }^{2}$. Ceci, bien sûr, obligeait à quitter les terres relativement bien cartographiées des grandes puissances européennes (Allemagne, France, Grande-Bretagne) pour s'aventurer dans des histoires nationales complexes, tortueuses, tourmentées, qui compliquent à l'infini la tâche du statisticien, mais enrichissent sans aucun doute l'ensemble d'information de l'économiste, et partant multiplient les sources de réflexion et d'inspiration.

\section{LES DETTES PUBLIQUES EUROPÉENNES, 1880-1914}

\section{Faits stylisés}

La figure 1, qui décrit l'évolution des ratios dette/PIB pour un large échantillon de pays européens, fait apparaître un certain nombre de traits marquants. D'abord, et peut-être surtout, l'étalon or, dont la réputation d'orthodoxie monétaire et financière est excellente, fut un système où le niveau des dettes publiques était considérable. On ne peut manquer d'être frappé par le fait qu'une majorité de pays européens avaient des ratios d'endettement public (dette/PIB) très élevés, généralement au-dessus du seuil de $60 \%$ et même bien souvent au-dessus de $80 \%$. Ce phénomène, qui caractérise les économies « du Sud » (Espagne, Italie, Grèce, Portugal), est aussi observé en France et aux Pays-Bas. Même le Royaume-Uni connaît au début de la période un endettement élevé (environ $60 \%$ ).

En termes de tendance, deux phases peuvent être identifiées ${ }^{3}$. La première, qui prend en fait sa source dans les années 1870, est caractérisée par un boom

1. Pour une critique du caractère excessivement anglo-centrique de la littérature sur le système international dans la période antérieure à l'émergence de l'étalon or (18481873), voir Flandreau [1995].

2. Pour un point de vue analogue, du côté réel, voir Crafts [1984].

3. Ce boom des dettes publiques européennes fut parfaitement identifié par les contemporains comme Baxter qui écrivait en 1871: «Les nations latines grèvent leur développement industriel par l'acharnement qu'elles mettent à s'endetter. La France ploie sous l'accroissement récent de son fardeau. L'Italie se retrouve presque écrasée. L'Espagne et le Portugal accroissent les obstacles sur le chemin de leur redressement commercial. L'Amérique latine accroît régulièrement ses impôts à la hauteur de ses dettes. En Europe de l'Est, l'Autriche continue d'emprunter pour financer ses dépenses militaires ; la Russie s'en va chargeant sa pauvre population; tandis que la Turquie et l'Égypte empruntent jusqu'à la banqueroute. Tous ces États sont en train de se disqualifier pour la course avec les industries anglo-saxonnes et teutonnes ». 
des dettes publiques. Cette tendance haussière est d'autant plus remarquable que l'on est dans une situation générale de paix. Échappent cependant à ce mouvement le Royaume-Uni et la majorité des «petites économies»: Belgique, pays scandinaves et Suisse ${ }^{1}$. À partir du milieu de la période (1896, environ), une décrue importante s'engage. Elle touche surtout les pays qui ont connu les accroissements les plus substantiels auparavant. En 1913, tous les pays de notre échantillon se retrouvent au-dessous du seuil de $100 \%$ et, pour une majorité, au-dessous de la barre des $60 \%$.

L'étalon or semble donc avoir été capable d'accommoder des niveaux de dettes extrêmement hétérogènes. En effet, s'il est vrai que les principales crises financières qui ont affecté l'étalon or (Espagne, Grèce, Portugal) touchent des pays ayant des ratios d'endettement élevés, plusieurs États extrêmement endettés traversent la période sans encombre. C'est le cas notamment de la France, mais aussi des Pays-Bas et, dans une certaine mesure, de l'Italie. Aussi, l'hétérogénéité des niveaux de dette tranche-t-elle avec l'extraordinaire convergence des taux longs. En effet, la figure 2 indique que la convergence des taux d'intérêt entre les pays de l'échantillon fut considérable, tous les taux se trouvant dans un intervalle d'un peu plus d'un point à partir de 1908 environ $^{2}$. Il ne semble donc pas qu'ait existé un lien clair entre le niveau des dettes et celui des taux. Par ailleurs, ce résultat « transversal » se retrouve aussi dans une approche longitudinale. Ainsi, la période antérieure à 1896, où les dettes publiques s'accroissent par rapport au PIB, est caractérisée par une décrue des taux nominaux, tandis que la période, où les dettes publiques refluent, s'accompagne d'une remontée des taux. Finalement, le niveau élevé des dettes publiques européennes et leur extrême hétérogénéité sont en contraste total avec la tendance lourde de convergence des taux longs.

On arrive ainsi à une conclusion tout à fait étonnante qui, à première vue, ne semble qu'épaissir l'énigme de la stabilité de l'étalon or, puisqu'il paraît tout à fait impossible de justifier cette stabilité en invoquant des motifs de modération financière. Au contraire, on serait tenté de renverser les termes de la causalité, pour suggérer que c'est la stabilité de l'étalon or qui aurait permis l'accumulation considérable des dettes publiques.

\section{Soutenabilité de la dette et profitabilité des dépenses d'infrastructure}

Ce constat soulève en ricochet le problème de la soutenabilité. Se peut-il que l'étalon or ait caché en filigrane un problème plus fondamental, celui d'une insoutenabilité à terme ? En fait, la tendance générale au déclin des ratios d'endettement après 1896 invite à répondre par la négative. Par ailleurs, le

1. La similitude des ratios de dette publique scandinaves est tout à fait remarquable et permet sans doute de comprendre pourquoi ces pays ont pu maintenir avec succès l'Union monétaire scandinave pendant tout la période. À titre de comparaison, on pourra opposer l'évolution des dettes publiques dans les quatre États membres de l'Union monétaire latine : France, Belgique, Italie et Suisse.

2. Force est de constater cependant que cette convergence semble s'être accompagnée de difficultés vers le milieu de la période, du moins pour les pays du Sud, ainsi que l'indique la hausse des taux espagnol, portugais et grec pendant les années 1890. 
Graphique 1. Ratios dette/PIB en Europe 1880-1914

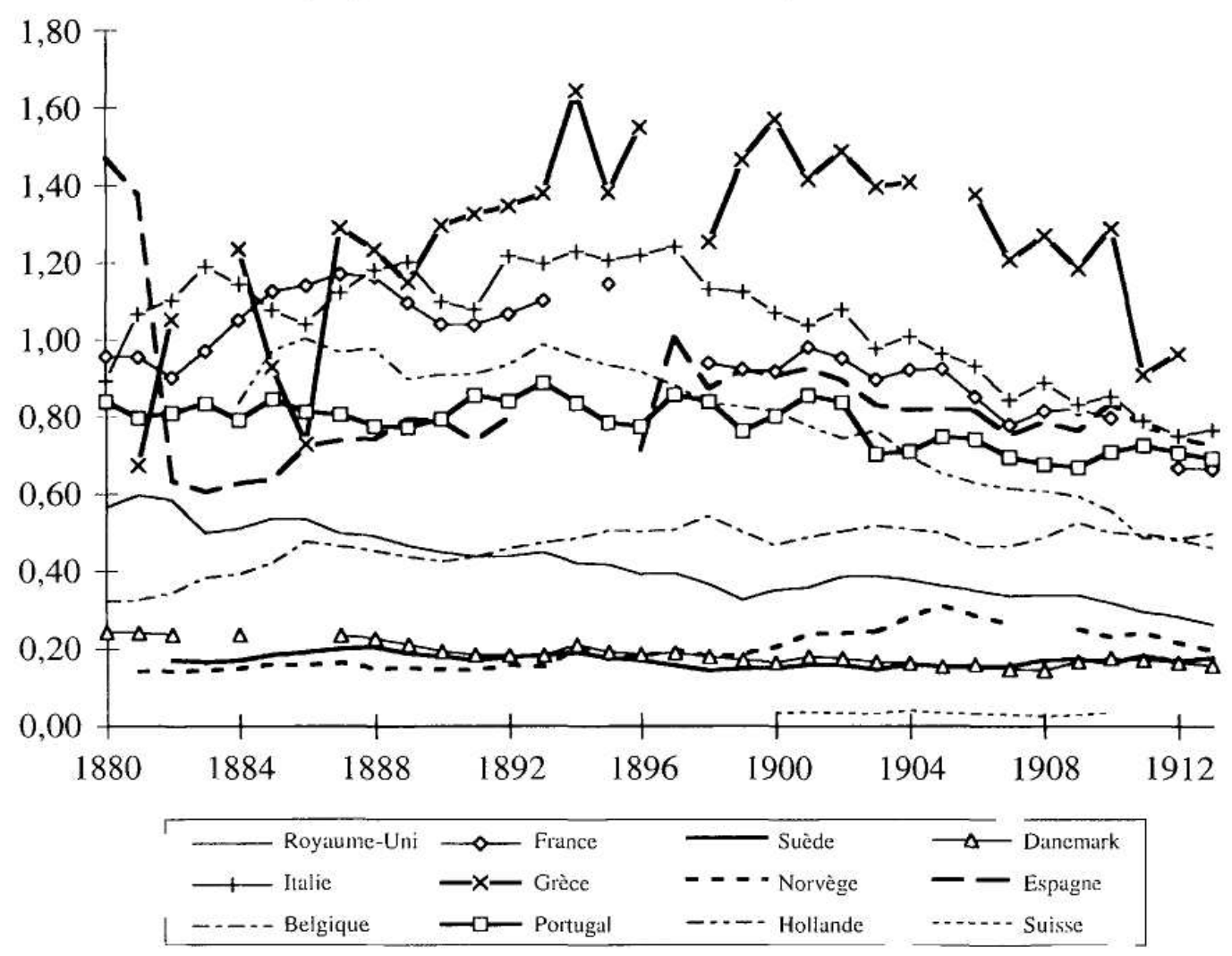

Graphique 2. Taux longs en Europe, 1880-1914

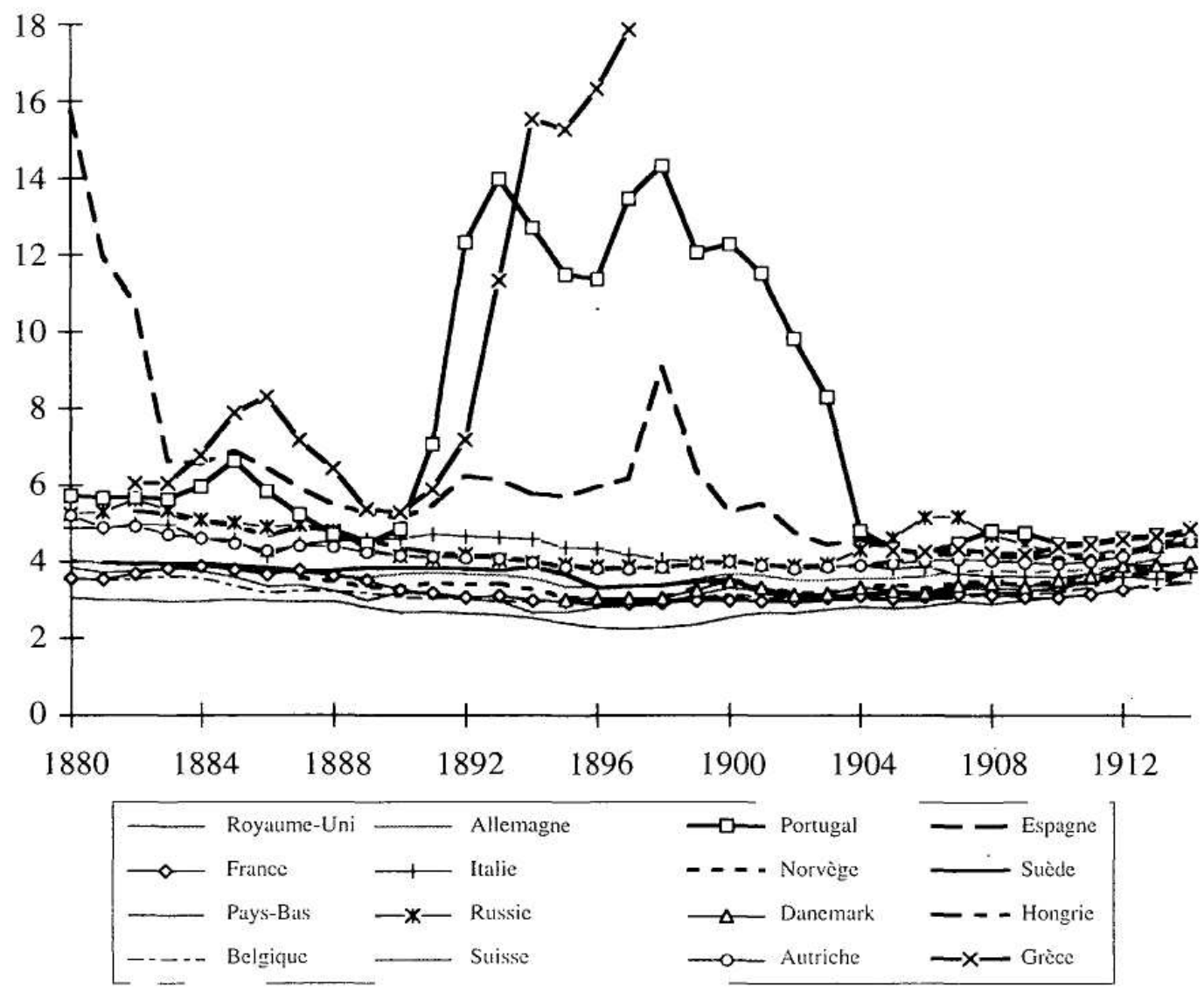


niveau élevé des dettes publiques n'est peut-être pas surprenant, si l'on garde à l'esprit que, contrairement à l'idée reçue, les États ont joué un rôle considérable dans le développement économique au XIX ${ }^{\mathrm{e}}$ siècle.

En effet, nous avons trouvé que, pour la quasi-totalité des pays considérés, les dettes publiques correspondaient, pour une fraction importante - voire dans certains cas pour la totalité -, à des investissements d'infrastructures, pris en charge par les pouvoirs publics. Ceci est particulièrement saillant pour les États allemands, où la quasi-totalité de la dette est constituée de railway debts, mais aussi pour les pays scandinaves, pour la Russie, pour l'Autriche-Hongrie, et, dans une moindre mesure, pour la France à la suite du plan Freycinet, etc. En fait, par rapport à cette tendance générale, c'est encore le Royaume-Uni qui fait figure d'exception, puisque son ratio d'endettement, qui décline régulièrement, n'est que le mauvais souvenir des obligations énormes contractées pendant les French wars au début du siècle, et que ce sont les capitaux privés qui ont financé son développement.

L'existence d'une « dette publique d'infrastructure » importante mitige donc sérieusement l'évaluation de la soutenabilité. D'abord, ces dépenses ont généralement donné lieu à la création d'actifs productifs dont le produit appartient à l'État, et qu'il faut donc déduire du service de l'intérêt sur les emprunts ayant permis de financer ces projets. Sous cet angle, de grandes différences peuvent être observées d'un pays à l'autre et il faudrait pouvoir cerner avec plus de rigueur l'écart entre dette brute et dette nette ${ }^{1}$. À un pôle, les États scandinaves ou certains États allemands équilibrent leurs comptes ou même gagnent de l'argent sur leurs investissements d'infrastructures. Il se peut même que certains États, qui, comme l'État fédéral suisse, ne se sont endettés qu'à des fins productives, soient en fait des créanciers nets et non des débiteurs nets. Dans ce cas, l'État s'est substitué à l'entreprise privée. À l'autre pôle, la Hongrie, l'Autriche ou la France ont nationalisé certaines lignes de chemins de fer, alors que celles-ci perdaient de l'argent, ce qui en retour grève les finances publiques ${ }^{2}$.

Même dans ce cas, cependant, il se peut que les dépenses d'infrastructure aient en fait contribué à améliorer la soutenabilité de la dette publique, par exemple lorsque les investissements ont favorisé le développement économique. Il semble bien, en effet, que tel ait été le cadre de raisonnement des investisseurs pour juger de la soutenabilité des dettes d'États très endettés. C'est ainsi par exemple qu'Alfred Neymarck déclarait en 1887 à propos du Portugal — avec il est vrai peut-être un peu trop de confiance et d'enthousiasme : "Il convient de faire remarquer que le capital emprunté par le gouvernement portugais depuis 1871 a été appliqué à la construction de chemins de fer, routes, postes, télégraphes, etc., ainsi qu'au développement et à l'amélioration des colonies. Tous les progrès accomplis n'ont pu être obtenus qu'en augmentant les charges de la dette, mais le Portugal ressent déjà les heureux effets de ces dépenses productives. Les recettes du Trésor augmentent progressivement et la conséquence naturelle est la diminution du déficit ${ }^{3}$ ». Finalement, on s'acheminerait ainsi vers

1. Il va sans dire que cet exercice est extrêmement difficile, puisqu'il implique de collecter une information détaillée sur les rendements de l'investissement productif.

2. Ainsi le Portugal cédera-t-il à ses créanciers, dans les années 1890, l'usufruit de ses manufactures de tabac, extrêmement profitables.

3. Neymarck [1887], p. 54-55. 
une explication « réelle » de la compatibilité entre l'importance des dettes publiques et la stabilité de l'étalon or, imputable à la profitabilité directe et indirecte des investissements que ces dettes avaient financés.

Ce type d'analyse n'est pas sans évoquer la thèse classique de Gerschenkron, suivant laquelle les nations appartenant aux générations successives de développement économique auraient utilisé un input public croissant, d'autant plus grand que le retard relatif d'une génération donnée par rapport aux nations déjà industrialisées était important. L'accumulation des dettes européennes refléterait alors des trajectoires nationales, et les fluctuations du rapport dette/PIB seraient l'image des vagues successives d'industrialisation. Tout en remettant le test rigoureux de cette proposition à un travail ultérieur, on peut d'ores et déjà souligner les limites d'une telle explication. En effet, le mouvement d'accroissement des dettes publiques identifié entre 1880 et 1914 inclut des pays ayant connu un processus d'industrialisation à des dates fort différentes ${ }^{1}$.

Il semble donc que l'évolution des dettes publiques aient été soumises à des facteurs communs, au moins pour partie. Sinon comment justifier l'existence d'un certain degré de cocyclicité des rapports dette/PIB, même pour des pays appartenant à des générations d'industrialisation fort différentes ?

\section{Le rôle de l'inflation?}

Les conclusions précédentes conduisent à chercher des déterminants communs dans la tendance haussière puis baissière des ratios dette/PIB. En fait, la coïncidence de ces mouvements avec ceux, bien connus, des prix sur longue période suggère une explication simple : l'alourdissement des dettes a coïncidé avec la baisse des prix, son allégement avec la hausse régulière de la Belle Époque.

D’un côté, la déflation mondiale d'avant-1896 aurait agi de façon mécanique dans le sens d'une augmentation des ratios d'endettement public, annulant ainsi une partie des effets positifs de la croissance de la période. À l'inverse, l'inflation d'après-1896 se serait superposée à la croissance dans l'amortissement de la dette publique.

Cette explication permettrait de comprendre les liens, en surface surprenant, entre l'évolution des ratios dette/PIB et celle des taux d'intérêt longs. En effet, on sait que le phénomène bien connu du «paradoxe de Gibson » s'est traduit par une corrélation positive entre les mouvements longs des prix et les taux d'intérêt nominaux. On obtient donc finalement un accroissement du poids des dettes quand les taux nominaux baissent et une réduction des ratios quand les taux nominaux montent.

Bien sûr, ces deux mouvements sont susceptibles de produire des mouvements opposés en termes de convergence ou de divergence des taux d'intérêt. En effet, l'alourdissement de la dette publique en termes du PIB finira par plonger les pays dont les finances publiques sont les moins bien organisées dans des crises sérieuses. De fait, la hausse des ratios d'endettement public jusqu'en

1. Il serait intéressant de clarifier la dynamique comparée de croissance de la dette publique et de l'économie, afin de mieux cerner le sens et la portée de la thèse de Gerschenkron. 
1896 culmine avec des difficultés dans les finances publiques de trois des six pays qui ont connu les hausses les plus marquées (Espagne, Portugal et Grèce). De façon intéressante, ces trois pays sont aussi les moins développés, et donc apparaissent comme les plus fragiles. Les crises des années 1890 seraient la résultante de deux facteurs, structurels d'un côté et nationaux de l'autre, puisque ce n'est pas n'importe quel sous-ensemble de pays qui plonge dans les difficultés. À l'inverse, l'inflation régulière de la Belle Époque aurait joué un rôle important dans la stabilité de l'étalon or. Finalement, derrière le secret de la stabilité « systémique » de la zone-or se cache peut-être, bien plus que la croissance, l'inflation.

\section{CRISES DE LA DETTE ET CONVERGENCE}

L'identification d'une combinaison de facteurs nationaux et internationaux dans les «pathologies », qui se développent à la fin de la longue déflation de 1873-1896, invite à réfléchir de façon plus approfondie sur la nature des crises qui ont affecté la «périphérie » européenne de l'étalon or, au cours des années 1890.

\section{Pathologies de l'étalon or, 1890-1902}

De façon générale, le développement des crises de la dette publique prend sa source dans une succession de difficultés budgétaires conduisant un pays donné à recourir systématiquement à l'emprunt étranger, libellé en devises or, auprès de l'un des principaux marchés du « centre », c'est-à-dire Paris ou Londres. Ces emprunts sont réalisés auprès d'un intermédiaire bancaire, qui souvent gère les relations du Trésor avec l'étranger (service des intérêts, émission de traites sur le Trésor, etc.). Cet intermédiaire se charge, le plus souvent en coopération avec un syndicat bancaire, de l'émission (placement et underwriting) de la dette extérieure.

Au fur et à mesure que cette dette s'accumule, cependant, les exigences de l'intermédiaire habituel, en termes de garanties, de transparence et de contrôle des comptes publics, et de retour prompt à l'équilibre, s'accroissent. Souvent, cet intermédiaire tente d'utiliser le rôle de « signalement » et de réputation que sa participation à l'émission lui confère, pour imposer ses vues. Ceci conduit le débiteur à chercher l'aide d'autres intermédiaires (généralement des maisons de second rang, ou moins bien implantées sur la place) qui, au prix d'un taux d'intérêt généralement fort élevé, acceptent de contribuer à la fourniture de crédits courts, et à leur éventuelle consolidation. Il se peut qu'à ce stade les finances soient remises en ordre : tel fut le cas de l'Autriche-Hongrie et de la Russie à la fin des années 1880, et de l'Espagne à la fin des années 1890. Cependant, il arrive que la détérioration se poursuive. Dans ce cas, la part de la dette publique à court terme augmentera régulièrement, jusqu'à la crise finale où les refinancements sont refusés. Le débiteur n'a alors plus d'autre choix que de suspendre le paiement des intérêts. Ce schéma général fut illustré par l'Espagne en 1882 et par la Grèce et le Portugal dans les années 1890.

\section{Procédures budgétaires et structures fiscales}

En dehors des conflits armés - la guerre hispano-américaine de 1898, le conflit gréco-turc de la même époque, la guerre russo-japonaise de 1904-1905 -, 
qui constituent des causes bien connues de dégradation de la situation financière des États, ainsi que des guerres civiles, il apparaît que l'une des caractéristiques communes à tous les pays qui connaissent des crises ou des difficultés plus ponctuelles au cours de la période 1880-1913 est de nature institutionnelle. Dans les pays d'Europe du Sud et en Russie, le contrôle des assemblées parlementaires sur l'élaboration du volet dépenses des budgets est, en effet, faible, voire inexistant, et, faute de procédures d'audit, l'exécution peut s'écarter sensiblement des engagements. En outre, on retrouve, dans la plupart de ces pays, des lacunes similaires dans les structures fiscales : élasticité faible des recettes à la croissance des revenus, en raison notamment de la prépondérance des impôts indirects d'accises et d'impôts directs souvent cédulaires, de l'absence d'un impôt progressif sur les revenus et d'insuffisances des systèmes de collecte et de contrôle fiscal. En pratique, force est de constater que les stabilisations réussies de la Russie ou de l'Autriche-Hongrie sont passées par des réformes profondes du système fiscal (régime d'imposition, audit des comptes publics, etc.).

Cette importance du processus fiscal dans la détermination de la stabilité monétaire peut d'ailleurs être illustrée dans le contexte des États à structure « fédérale », comme l'Allemagne ou l'Empire austro-hongrois : dans les deux cas, la faiblesse des ressources fiscales propres du centre et la difficulté d'obtenir des États fédérés des contributions suffisantes pèsent sur l'équilibre financier des budgets centraux. C'est ainsi que l'Empire allemand doit payer jusqu'en 1914 une prime « politique » lorsqu'il emprunte sur les marchés internationaux, qui lui facturent un taux de près d'un point supérieur à ceux pratiqués pour la France ou la Grande-Bretagne.

\section{Des effets " systémiques "?}

Les dates auxquelles surviennent les principales crises financières et leur géographie suggèrent l'existence possible de facteurs «systémiques » liés aux choix de portefeuille des grands investisseurs internationaux et à leurs pratiques d'analyse du « risque-pays ». D'une part, en effet, il apparaît que les difficultés financières des pays du Sud se manifestent après la résolution des problèmes dans les Empires - et singulièrement en Russie - et leur stabilisation, une réorientation des flux de financement internationaux s'opérant alors au profit de ces États, et au détriment de l'Europe du Sud. D'autre part, le développement, par les grands établissements financiers anglais et français, de techniques « modernes » d'analyse du risque-pays, avec une classification des pays en catégories, est susceptible d'avoir favorisé une certaine contagion, un « effet Tequila » avant la lettre. La figure 2 montre bien que le début des années 1890 est caractérisé par une hausse violente et concomitante des taux longs en Espagne, en Grèce et au Portugal, hausse qui coïncide avec des mouvements similaires dans plusieurs pays d'Amérique latine, ainsi qu'avec les difficultés de la Banque Baring au Royaume-Uni (voir Kindleberger [1985]).

\section{La résolution des crises}

En dépit de la gravité des crises d'endettement recensées et d'un ostracisme initial évident des prêteurs internationaux à l'égard des États affectés, la convergence des taux s'opère en quelques années. Les trois pays du Sud (Espagne, Grèce, Portugal) qui connaissent les difficultés les plus sérieuses dans les années 1890 illustrent trois configurations possibles. 
L'Espagne, moins touchée que les deux autres pays, réussit à piloter à vue dans les années 1890. La guerre de 1898 avec les États-Unis, à l'issue de laquelle l'Espagne perdra les derniers restes de son empire colonial, débouche sur une forme de défaut partiel par lequel les agents espagnols sont contraints de changer leurs créances or en créances papier. Ce mécanisme, qui s'inspire de la méthode des affidavits développée par les Italiens plus de vingt ans plus tôt, a l'avantage de doter le gouvernement d'un coussin amortisseur. Lorsque la monnaie se déprécie, le service des intérêts s'allège, et facilite donc la gestion de la dette. Avec la moitié de sa dette externe «convertie» en monnaie interne, l'Espagne pourra limiter la dépréciation de sa monnaie jusqu'en 1914.

Le cas grec, en revanche, se présente comme une mise en ordre drastique des finances publiques, imposée par l'extérieur : c'est la «thérapie de choc». Les créanciers étrangers - notamment la France et l'Angleterre, puissances garantes de la dette publique grecque - , qui, par l'intermédiaire de leurs États, avaient dû supporter le paiement de certains coupons à leurs détenteurs, imposent la création d'une société privée sous contrôle international qui prend en charge la collecte des impôts et le paiement des intérêts sur une base minimale, les excédents étant répartis entre les créanciers $(60 \%)$ et l'État grec $(40 \%)$. Très vite, les surplus s'accumulent permettant à la Grèce dès 1904 un retour sur les marchés internationaux, ainsi que la convergence des taux. Par ailleurs, la réforme fiscale et la croissance économique induisent un allégement de la charge de la dette.

Le cas portugais, enfin, représente un troisième cas de figure. La crise initiée en 1890 traîne en longueur pendant toute la décennie, au fur et à mesure que les différentes solutions proposées butent sur les rivalités des créanciers. Le Portugal se verra même fermer par les autorités publiques françaises l'accès au marché parisien pendant quelques années. C'est finalement le Convenio de 1902 qui viendra mettre un terme à de longues négociations : techniquement, il s'agit d'un défaut, ce qui vaudra au gouvernement portugais la méfiance des investisseurs. Comme l'Espagne, cependant, le Portugal conserve jusqu'à la fin de la période des finances fragiles, ainsi que l'indique la forte proportion du service de la dette dans les dépenses totales.

\section{CONCLUSION}

Au cours de la période 1880-1914, les tendances générales à la stabilisation, puis à la résorption des ratios d'endettement public dans la plupart des pays européens membres du système monétaire international d'étalon or, la convergence, marquée en fin de période, de leurs taux d'intérêt longs et les caractéristiques des crises d'endettement et de leurs modes de résolution suggèrent quelques pistes de recherche qui pourraient permettre de mieux cerner la nature de l'étalon or, ainsi que les mécanismes ayant assuré la cohésion de cette zone monétaire fortement décentralisée.

Quatre hypothèses se détachent. D’un point de vue systémique, il semble que le taux d'inflation global ait joué un rôle important soit en fragilisant (18801896), soit en affermissant (1896-1913) la zone or. En second lieu, le rôle des flux internationaux de capitaux, leur montant et leur structure peuvent avoir 
servi de courroie de transmission aux déstabilisations des années 1890. Dans un marché globalisé, les problèmes de finances publiques d'un pays donné peuvent avoir des conséquences considérables pour la stabilité d'autres pays. En conséquence, les mécanismes qui assurent l'orthodoxie financière sont encore plus importants dans une économie mondialisée que dans une économie segmentée. Ceci conduit à notre troisième point, qui renvoie aux processus de contrôle des finances publiques : en d'autres termes, il faudrait pouvoir approfondir l'évolution de l'organisation du processus budgétaire dans les pays concernés, son éventuelle réforme, et la possible convergence vers un modèle uniforme ainsi que l'a suggéré Théret [1994]. Enfin, il faudra clarifier l'articulation entre dettes publiques et croissance économique, de façon à éclairer les fondements « réels » de la stabilité du gold standard, édifice qui fut capable d'accommoder des dettes publiques extrêmement élevées.

\section{RÉFÉRENCES BIBLIOGRAPHIQUES}

BaXter, Dudley R. [1871], National Debts, $2^{\mathrm{e}}$ éd., Londres, Robert John Bush.

CrafTS, N. [1984], "Patterns of Economic Development in Nineteenth Century Europe », Oxford Economic Papers, 36, p. 438-58.

FLANDREAU M. [1995], L'or du monde. La France et la stabilité du système monétaire international, 1848-1873, Paris, L'Harmattan.

FLANDREAU M., LE CACHEUX J. [1996], « La convergence est-elle nécessaire à la création d'une zone monétaire ? Réflexions sur l'étalon or 1880-1914», Revue de l'OFCE, 58 , juillet.

KINDLEBERGER, Charles P. [1985], Keynesianism versus Monetarism and other Essays in Financial History, Londres, Allen and Unwin.

Maddison, A. [1991], Dynamic Forces in Capitalist Development, Oxford, Oxford University Press.

NeymarCK, A. [1887], Les dettes publiques européennes, Paris, Guillaumin.

THÉRET B. [1995], «Régulation du déficit budgétaire et croissance des dépenses de l'État en France de 1815 à 1939 », Revue économique, 46 (1), p. 57-90. 